\section{NP-011 MEDICATION RECONCILIATION IN AN EMERGENCY DEPARTMENT - PROCESS ASSESSMENT FOR A MORE EFFICIENT SERVICE}

M Dreetz Holt, A Teigen, M Hermann, R Kjome. Stavanger University Hospital, Stavanger, Norway

\subsection{6/ejhpharm-2020-eahpconf.469}

Background and importance Medication reconciliation (MR) is the process of providing an exact and accurate list of all medications a patient is taking. This process is necessary to ensure that patients get the correct medications when admitted to hospital, thus preventing drug related problems. The pharmacists in the emergency department at Stavanger University Hospital use a method based on IMM-methodology (Integrated Medicines Management) when executing MR. This is time consuming, partly due to asking patients open questions and the lack of electronic resources available at the time IMM-methodology was developed.

Aim and objectives This thesis aims to make the pharmacist's method for MR at the emergency department more efficient, thus obtaining correct medicine lists as early as possible for more patients.

Materials and methods An observation study surveyed possible improvements in the established method for MR at Stavanger University Hospital. This subsequently led to implementing a revised method for MR through an intervention study, comparing the methods with efficiency (time usage/patient) and quality (proportion of patients with discrepancies and number of discrepancies per patient). The revised method was deemed not qualitatively inferior to the established method if the proportion of discrepancies had a maximum deviation of $10 \%$.

Results In total two hundred patients (78 years $\pm 10,58 \%$ women) were included in the control group and currently hundred patients (78 years $\pm 9,50 \%$ women) in the intervention group. The time usage for completing a MR in the intervention group was reduced by $34 \%$ compared to the control group. There was no difference in the proportion of patients with discrepancies/number of discrepancies per patient (respectively 79\%/1.9 in the control group and 80\%/1.9 in the intervention group).

Conclusion and relevance Data from the first 100 patients in the intervention group shows that the revised method for performing MR makes the process more efficient without significantly deterioration of quality.

\section{NP-012 COST EFFECTIVENESS ANALYSIS AND INDIRECT COMPARISON CAN SUPPORT THE PRICE' DEFINITION OF A DRUG? THE CASE OF ENCORAFENIB AND BINIMETINIB IN METASTATIC MELANOMA, THE PHARMACIST'S POINT OF VIEW}

${ }^{1} \mathrm{~A}$ Marinozzi, ${ }^{1} \mathrm{AMP}$ Mangano, ${ }^{1} \mathrm{E}$ Antonicelli, ${ }^{1} \mathrm{~A}$ Caprodossi, ${ }^{1} \mathrm{R}$ Connestari, ${ }^{1} \mathrm{M}$ Lalli, ${ }^{1}$ E Maradonna, ${ }^{2} \mathrm{R}$ Berardi, ${ }^{1} \mathrm{~L}$ Patregnani. ${ }^{1} \mathrm{PF}$ Farmaceutica - ARS - Regione Marche, Ancona, Italy; ${ }^{2}$ AOU Ospedali Riuniti di Ancona - Clinica Oncologica, Ancona, Italy

\subsection{6/ejhpharm-2020-eahpconf.470}

Background and importance The hospital pharmacist (HP) plays a key role in accessing new drugs at both regional and hospital level.

Aim and objectives The aim of this study is to propose a costeffective approach, from the perspective of the HP, comparing the case of Encorafenib and Binimetinib, (EB) two BRAF metastatic melanoma treatments (soon to be commercialized), with similar competitor drugs.

Materials and methods We developed a probabilistic model and a survey to quantify the economic impact of the price of these two new drugs. A cost-effectiveness model (in terms of OS) was developed using Montecarlo's simulations comparing EB vs Dabrafenib-Trametenib (DT) vs Vemurafenib-Cobimetinib (VC): The comparison arm of the 3 drugs registration studies was the Vemurafenib as the results of the arms were similar (both PFS and OS). In order to estimate the potential cost of EB (currently in negotiation), a survey was conducted within a Regional University Hospital taking into account Clinicians, HPs and Economists point of view.

Results The survey showed that EB could cost up to $18 \%$ more than DT, due to the 7.5 -months of life gained (MoLG) more than the best competitor. The price emerged from the survey was used to conduct the cost-effectiveness analysis in order to estimate an incremental relationship between the alternatives. From the 10,000 simulations carried out considering a threshold value of $€ 5,000 / \mathrm{MoLG}$ emerged that $\mathrm{EB}$ was cost effective in $80 \%$ vs. DT showing an incremental ratio of $€ 4,239 /$ MoLG; Furthermore, it was found to be cost effective in $83 \%$ vs.VC showing an incremental ratio of $€$ 3,129/MoLG.

Conclusion and relevance EB potential cost could be more than the alternatives, but with a negotiation price similar to the available alternatives, the NHS would benefit in terms of health (MoLG) without significant additional expense. Furthermore, when direct comparisons are not available, it is advisable to analyze possible strategies of indirect comparison such as the Network metaanalysis. The methodology used in the survey to investigate the HP's willingness to pay could be a support tool that could be used from AIFA representatives to assess the perception of added value in the analysis of new therapeutic treatments.

\section{NP-013 THE NEOCHECK PROJECT: DEVELOPMENT OF A PRESCRIPTION-SCREENING TOOL SPECIFIC TO NEONATOLOGY}

${ }^{1,2}$ Thomas Rudolf von Rohr, ${ }^{3}$ Roberta de Luca, ${ }^{1,2}$ Pascal Bonnabry, ${ }^{3}$ Riccardo Pfister, ${ }^{1,3}$ Caroline Fonzo-Christe. 'Geneva University Hospitals, Pharmacy, Geneva, Switzerland; ${ }^{2}$ School of pharmaceutical sciences, University of Geneva, University of Lausanne, Geneva, Switzerland; ${ }^{3}$ Geneva University Hospitals, Neonatology and Pediatric Intensive Care Unit, Geneva, Switzerland

\subsection{6/ejhpharm-2020-eahpconf.471}

Background and importance Neonatal pharmacotherapy is challenging and often based on little evidence. Off label use of drugs is common practice and patients are at a high risk of medication errors and drug-related problems. Prescriptionscreening tools are used in geriatrics, internal medicine and pediatrics to optimize drug prescribing.

Aim and objectives Our aim was to develop a prescriptionscreening tool specific to neonatology.

Materials and methods Clinical guidelines on neonatal pharmacotherapy were identified by a literature review and synthetized into short statements. A 2-rounds Delphi consensus method was used to establish the content validity of Neocheck. The statements were submitted to a group of 23 experts in 10 Swiss neonatology centers. The level of agreement was evaluated on a 5-point Likert scale ( 1 being the highest level of agreement). Statements for which $>65 \%$ of 